\title{
Reversible Harmonic Maps between Discrete Surfaces
}

\author{
DANIELLE EZUZ, Technion - Israel Institute of Technology \\ JUSTIN SOLOMON, Massachusetts Institute of Technology \\ MIRELA BEN-CHEN, Technion - Israel Institute of Technology
}

Information transfer between triangle meshes is of great importance in computer graphics and geometry processing. To facilitate this process, a smooth and accurate map is typically required between the two meshes. While such maps can sometimes be computed between nearly isometric meshes, the more general case of meshes with diverse geometries remains challenging. We propose a novel approach for direct map computation between triangle meshes without mapping to an intermediate domain, which optimizes for the harmonicity and reversibility of the forward and backward maps. Our method is general both in the information it can receive as input, e.g., point landmarks, a dense map, or a functional map, and in the diversity of the geometries to which it can be applied. We demonstrate that our maps exhibit lower conformal distortion than the state of the art, while succeeding in correctly mapping key features of the input shapes.

CCS Concepts: • Computing methodologies $\rightarrow$ Shape analysis;

Additional Key Words and Phrases: Shape analysis, shape matching, shape correspondence, harmonic maps

\section{ACM Reference format:}

Danielle Ezuz, Justin Solomon, and Mirela Ben-Chen. 2019. Reversible Harmonic Maps between Discrete Surfaces. ACM Trans. Graph. 38, 2, Article 15 (March 2019), 12 pages.

https://doi.org/10.1145/3202660

\section{INTRODUCTION}

Mapping 3D shapes to one another is a basic task in computer graphics and geometry processing. Correspondence is needed, for example, to transfer artist-generated assets such as texture and pose from one mesh to another (Sumner and Popović 2004), to compute in-between shapes using shape interpolation (Heeren et al. 2012; Von-Tycowicz et al. 2015), and to carry out statistical shape analysis (Munsell et al. 2008). In these applications, desirable correspondences satisfy some basic key properties: they should be smooth to avoid introducing geometric noise during transfer; they should preserve semantic features to ensure that key features are

D. Ezuz acknowledges funding from the Irwin and Joan Jacobs fellowship. J. Solomon acknowledges the generous support of Army Research Office grant W911NF-12-R0011 ("Smooth Modeling of Flows on Graphs"), of National Science Foundation grant IIS-1838071 ("BIGDATA:F: Statistical and Computational Optimal Transport for Geometric Data Analysis"), from the MIT Research Support Committee, from an Amazon Research Award, from the MIT-IBM Watson AI Laboratory, and from the SkoltechMIT Next Generation Program. M. Ben-Chen acknowledges funding from the European Research Council (ERC starting grant no. 714776 OPREP) and the Israel Science Foundation (grant no. 504/16).

Authors' addresses: D. Ezuz, Technion - Israel Institute of Technology; email: danielle. ezuz@gmail.com; J. Solomon, Massachusetts Institute of Technology, 32 Vassar St, Cambridge, MA, 02139, USA; email: jsolomon@mit.edu; M. Ben-Chen, Technion Israel Institute of Technology; email: mirela@cs.technion.ac.il.

Permission to make digital or hard copies of part or all of this work for personal or classroom use is granted without fee provided that copies are not made or distributed for profit or commercial advantage and that copies bear this notice and the full citation on the first page. Copyrights for third-party components of this work must be honored. For all other uses, contact the owner/author(s).

(C) 2019 Copyright held by the owner/author(s).

0730-0301/2019/03-ART15

https://doi.org/10.1145/3202660 put in correspondence; and they should be reversible, namely, invariant to which of the two shapes is chosen as the source.

Many existing approaches to shape mapping focus on generating maps with low global distortion (e.g., preserving pairwise distances (Sahillioğlu and Yemez 2011)) at the expense of large local distortion, which reduces the quality of the correspondence and hinders downstream applications. On the other hand, approaches that minimize local distortion measures mostly require an intermediate domain and construct the final map as a composition through this domain (e.g., Aigerman and Lipman (2016)). While such methods minimize distortion of the maps into the intermediate domain, the distortion of the composed map can be large. This problem is exacerbated when the input shapes have significantly different geometric features, such as four-legged animals with different dimensions, e.g., a cat and a giraffe. In this case, the isometric distortion of the optimal map is expected to be large, and thus minimizing the distortion of the two maps into an intermediate domain is quite different from minimizing the distortion of the composition.

We propose a novel approach for computing a smooth and reversible map between surfaces that are not isometric to each other, without requiring an intermediate domain. We incorporate semantic information by starting from some user guidance given in the form of sparse landmark constraints or a functional correspondence. Our main contribution is the formulation of an optimization problem whose objective is to minimize the geodesic Dirichlet energy of the forward and backward maps while maximizing their reversibility. We compute an approximate solution to this problem using a high-dimensional Euclidean embedding and an optimization technique known as half-quadratic splitting (Geman and Yang 1995). We demonstrate that our maps have considerably lower local distortion than those from state-of-the-art methods for the difficult case of nonisometric deformations. We further show that our maps are semantically accurate by measuring their adherence to self-symmetries of the input shapes, their agreement with ground truth when the deformation is known, and their compatibility with human-generated segmentations.

\subsection{Related Work}

The shape correspondence literature is vast, and we refer the reader to recent surveys for a thorough review of the state of the art in geometry-driven (Tam et al. 2013; Van Kaick et al. 2011) and data-driven (Xu et al. 2016) shape correspondence. We will focus our related work overview on methods for computing maps between triangular meshes that can handle shapes that are far from being related by an isometric deformation. In this realm, we characterize methods by the type of input they require and the type of output they generate. We therefore distinguish between vertexto-vertex maps that yield a correspondence between the source and target vertices of the triangular meshes, precise maps that map 
every vertex on the source to a point on the triangulated surface of the target, and generalized maps that put in correspondence functions or probability distributions.

Fully Automatic Methods. Kim et al. (2011) suggested one of the first fully automatic methods for nonisometric shape matching that consistently generated high-quality outputs on a benchmark of shapes. This approach, denoted by BIM, generates a precise map as a blending of conformal maps, with blending weights optimized to minimize isometric distortion. While providing excellent results in many cases, BIM is limited to genus-zero surfaces and can introduce large distortions for some shapes. Recently, Zheng et al. (2017) suggested to map between high-genus surfaces with the same genus by decomposing the surfaces using a pants decomposition and then computing harmonic maps between a set of intermediate cylindrical domains. This leads to a piecewise harmonic map between the input surfaces, which is further relaxed using geodesic heat flow. While this approach can be used without user intervention, if a globally semantic map is needed, then accurate input landmarks are required, and it is limited to shapes of the same genus. Finally, Lähner et al. (2017) suggested a method that computes vertex-to-vertex maps based on a set of matching descriptors and pairwise distances. While the method is robust to topological changes, it may leave areas unmapped and is therefore less appropriate for applications such as texture transfer.

The functional map approach, introduced by Ovsjanikov et al. (2012), was originally designed for nearly isometric shapes but has since been extended to nonisometric matching (Burghard et al. 2017; Kovnatsky et al. 2013, 2015). This method generates a generalized map that puts in correspondence the function spaces on the mapped shapes. Nogneng and Ovsjanikov (2017) recently suggested a method that computes functional maps using fewer input descriptors by formulating commutativity constraints, and Huang and Ovsjanikov (2017) suggested to use adjoint functional maps to improve and analyze correspondences. Using a hybrid approach, Maron et al. (2016) optimize jointly for a functional map and sparse correspondences, which are then extended to a dense vertexto-vertex map. Finally, Solomon et al. (2016) compute a generalized map, which puts in correspondence probability distributions on the input shapes by minimizing the Gromov-Wasserstein distance between the shapes.

While generalized maps are beneficial for challenging mapping problems, such as mapping between shapes of different genuses, extracting a precise map is a necessary postprocessing step if the output map is to be used for transferring high-frequency data such as textures, normals, or deformations. Furthermore, when the shapes are geometrically different and the optimal map is not expected to be isometric, the shape correspondence problem is ill posed without additional semantic information. Such information can be given in the form of landmark constraints or an initial generalized map, from which a refined dense map can be computed.

Input: Landmarks. Parameterization-based approaches compute bijective smooth maps to a common intermediate domain and define precise maps between arbitrary shapes as the composition of the maps to the common domain. A variety of intermediate domains have been used in the literature, e.g., the plane (Aigerman et al. 2015), the sphere ( $\mathrm{Gu}$ et al. 2004), the hyperbolic disk
(Shi et al. 2017), and orbifolds (Aigerman et al. 2017; Aigerman and Lipman 2015, 2016; Tsui et al. 2013), to mention a few. These methods optimize the distortion of the map from the shapes to the target domain, but the composed map is not guaranteed in general to have low distortion. Furthermore, mapping through an intermediate domain places a topological restriction on the type of mapped shapes, as they should be topologically equivalent. Alternatively, Panozzo et al. (2013) compute a direct map between two triangle meshes without requiring an intermediate domain. Their method computes on-surface barycentric coordinates with respect to the source landmarks and then uses them with respect to the target landmarks to compute the corresponding point on the target shape. Similar to our approach, they use a high-dimensional Euclidean embedding to speed up the computation. Despite excellent results, in some cases a large number of landmarks are required to compute a correct correspondence. More recently, Mandad et al. (2017) use landmarks or extrinsic alignment for initialization and then optimize simultaneously for a generalized map and a precise map. We compare with their approach and demonstrate that our maps achieve better conformal distortion and adherence to the shape semantics.

Input: Generalized Maps. Several methods for recovery of vertex-to-vertex maps from generalized maps have been suggested. Shtern and Kimmel (2014) suggest a refinement technique that is based on heat kernel alignment, Rodolà et al. (2015) use a probabilistic model, and Vestner et al. (2017) solve a linear assignment problem. In general, vertex-to-vertex maps have higher conformal distortion than precise maps. Alternatively, Ezuz and Ben-Chen (2017) suggest a pointwise recovery method that generates precise maps using a smoothness prior, based on a spectral approach. Our method does not rely on spectral representations and thus exhibits fewer artifacts in complex cases.

\subsection{Contributions}

We present an algorithm for shape correspondence between nonisometric triangular meshes that has the following advantages:

- The algorithm is widely applicable, and the resulting maps are semantic and exhibit low conformal distortion.

- The formulation is simple and efficient to optimize and thus can be combined with additional energy terms and various initializations.

- The maps are accurate enough for downstream applications, such as shape interpolation and quad mesh transfer.

\section{BACKGROUND: HARMONIC MAPS AND LOCAL DISTORTION}

Suppose $M_{1}, M_{2} \subseteq \mathbb{R}^{3}$ are smooth, compact surfaces with or without boundaries. Given a map $\phi_{12}: M_{1} \rightarrow M_{2}$ from one into another, a natural task is to measure the distortion of $M_{1}$ as it is mapped via $\phi_{12}$ onto $M_{2}$; this distortion measure eventually will serve as an objective function for optimization problems whose unknown is the correspondence $\phi_{12}$. The basic role of these distortion measures is to evaluate whether nearby points are mapped to nearby points under $\phi_{12}$, at least differentially, a common proxy for the quality of the map. 
In the theory of differential geometry, a key distortion measure is the Dirichlet energy $E[\cdot]$ (defined below) of $\phi_{12}$; minimizers of $E[\cdot]$ are called harmonic maps. Intuitively, if we think of $M_{1}$ as a rubber sheet, a harmonic map represents an equilibrium position of the sheet after stretching it over $M_{2}$ and letting it compress. The Dirichlet energy and its minimizers find many roles in the geometry processing literature, most prominently in surface parameterization (Lévy et al. 2002), due to its intuitive measurement of distortion and connections to notions of conformality. At the same time, theory and practice of harmonic mapping become considerably more challenging when $M_{2}$ has areas of positive curvature; intuitively, these can cause the rubber sheet to slip or bunch, yielding singularities in gradient flow procedures designed to uncover harmonic maps.

In this section, we describe the basic construction of the Dirichlet energy and point out its advantages and flaws in the context of surface-to-surface correspondence; we also provide basic constructions for approximating the Dirichlet energy of a map between discrete surfaces. In Section 3, we then propose a modified notion of harmonicity designed to avoid singularities and asymmetry in the surface-to-surface correspondence pipeline.

\subsection{Smooth Surfaces}

Following Nishikawa (2000) and Urakawa (1993), harmonic maps between smooth surfaces are defined as the critical points of the Dirichlet energy:

$$
E\left[\phi_{12}\right]:=\frac{1}{2} \int_{M_{1}}\left|\mathrm{~d} \phi_{12}\right|^{2} d v_{1},
$$

where $\mathrm{d} \phi_{12}$ is the map differential and $d v_{1}$ is the volume element of $M_{1} . E\left[\phi_{12}\right]$ measures the total stretch of $M_{1}$ after it is warped onto $M_{2}$, as measured by the integrated norm of the Jacobian $\mathrm{d} \phi_{12}$. Formally, given an orthonormal basis $\left\{e_{1}, e_{2}\right\}$ for $T_{p} M_{1}$ at $p \in M_{1}$, the integrand can be expanded as

$$
\left|\mathrm{d} \phi_{12}\right|^{2}=\sum_{i=1}^{2}\left\langle\mathrm{~d} \phi_{12}\left(e_{i}\right), \mathrm{d} \phi_{12}\left(e_{i}\right)\right\rangle_{g_{2}\left(\phi_{12}(p)\right)},
$$

where $g_{2}$ is the metric of $M_{2}$.

Existence, uniqueness, and regularity of harmonic maps given assumptions on the geometry/topology of $M_{1}$ and $M_{2}$ as well as the homotopy class of $\phi_{12}$ are key themes in the twentieth-century differential geometry literature. A landmark paper by Eells and Sampson (1964) proves the existence of a harmonic map in each homotopy class under the assumption that $M_{2}$ has nonpositive curvature. The proof technique in that paper is attractive from a computational perspective: essentially they start with an arbitrary map in the prescribed homotopy class and use an analog of gradient descent to decrease the Dirichlet energy.

A key drawback of the Eells and Sampson proof technique from a computational perspective, however, highlights an issue with harmonic correspondence in the context of algorithmic mapping between surfaces. In particular, their gradient descent procedure can fail when the target $M_{2}$ has regions of positive curvature. Roughly, this singular behavior is explained by the fact that the objective $\left|\mathrm{d} \phi_{12}\right|^{2}$ is minimized globally by $\mathrm{d} \phi_{12} \equiv 0$, the constant map! This observation highlights the difference between harmonic mapping and elastic models like the ones proposed in Chao et al.

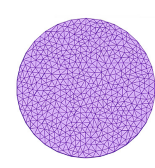

(a)

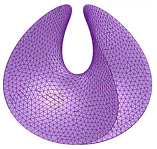

(b)

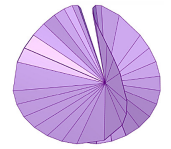

(c)

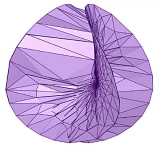

(d)

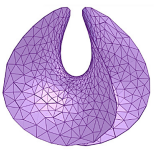

(e)
Fig. 1. Limitations of the piecewise linear discretization of the Dirichlet energy. (a) Source $M_{1}$ : a flat disk embedded in $\mathbb{R}^{3}$. (b) Target $M_{2}$ : enneper. (c) Initial piecewise linear map. (d,e) Final maps that minimize the energies in Equation (3) and Equation (4), respectively. See the text for details.

(2010) and Sorkine and Alexa (2007), which seek $\mathrm{d} \phi_{12}$ to be close to a rotation matrix rather than to the zero matrix. We will address this issue in our "reversible harmonic" formulation by adding the Dirichlet energy of $\phi_{12}^{-1}$ for the case of diffeomorphic correspondence; this has the added benefit of making forward maps $\phi_{12}$ and reverse maps $\phi_{21}$ critical points of the same objective function.

\subsection{Triangle Meshes}

For surfaces that are discretized as triangle meshes $M_{i}$, represented by their vertex, edge, and face sets $\left(\mathcal{V}_{i}, \mathcal{E}_{i}, \mathcal{F}_{i}\right)$, a pointwise vertex map $\phi_{12}$ assigns a point on a face of $M_{2}$ to each vertex of $M_{1}$. The extension of the vertex map to the interior of faces of $M_{1}$ determines the corresponding Dirichlet energy.

If the map is assumed to be affine on every face $f \in \mathcal{F}_{1}$, then the Dirichlet energy is given by

$$
E\left[\phi_{12}\right]=\frac{1}{2} \sum_{f \in \mathcal{F}_{1}}\left|\mathrm{~d} \phi_{12}(f)\right|_{2}^{2} a_{f},
$$

where $\mathrm{d} \phi_{12}(f) \in \mathbb{R}^{2 \times 2}$ is the unique linear transformation between $f$ and its image triangle $\phi_{12}(f)$, and $a_{f}$ is the area of $f$ (Pinkall and Polthier 1993). Equivalently, the energy can also be written as

$$
E\left[\phi_{12}\right]:=\frac{1}{4} \sum_{(u, v) \in \mathcal{E}_{1}} w_{u v}\left\|\phi_{12}(u)-\phi_{12}(v)\right\|_{2}^{2},
$$

where $w_{u v}$ is the cotangent weight of the edge $(u, v)$. This energy is convex and quadratic in the images of the vertices of $M_{1}$ and is therefore straightforward to minimize efficiently when $\phi_{12}$ is unrestricted, e.g., for planar parameterization (Lévy et al. 2002).

When $M_{2}$ is a non-Euclidean space, $\phi_{12}$ should be restricted to lie on $M_{2}$, leading to a constrained optimization problem that is harder to solve. In addition, a more serious issue is the linearity assumption itself. When $\phi_{12}$ does not sample the target surface $M_{2}$ well, the linear extension of $\phi_{12}\left(\mathcal{V}_{1}\right)$ can be far from $M_{2}$. In this case, minimizing the Dirichlet energy of the piecewise-affine map can lead to incorrect results.

Consider, for example, as in Figure 1, mapping a disk $M_{1}$ (a) to an enneper surface $M_{2}$ (b) with Dirichlet boundary conditions; since the target has negative curvature, in the smooth case (Eells and Sampson 1964), gradient flow will reach a harmonic map $\phi_{12}: M_{1} \rightarrow M_{2}$. An initial map (c) maps all the interior vertices of $M_{1}$ to a single interior vertex on $M_{2}$, and the boundary of the disk is mapped to the boundary of the enneper. Minimizing Equation (3) using gradient descent, the analog of Eells and Sampson's heat flow, with the side constraint that $\phi_{12}\left(\mathcal{V}_{1}\right)$ is restricted to lie on $M_{2}$ leads to a map (d) that is clearly not smooth. Effectively, 
Equation (3) aims to place the image of every vertex of $M_{1}$ in the Euclidean weighted average of the image of its neighbors. When the affine map samples the target poorly, this strategy fails to generate an approximation of a smooth map.

Alternatively, Izeki and Nayatani (2005) suggest an intrinsic formulation, the geodesic harmonic energy, which replaces the Euclidean distances in Equation (3) with the geodesic distances $d_{M_{2}}(\cdot, \cdot)$, as follows:

$$
E_{D}\left[\phi_{12}\right]:=\sum_{(u, v) \in \mathcal{E}_{1}} w_{u v} d_{M_{2}}^{2}\left(\phi_{12}(u), \phi_{12}(v)\right) .
$$

As shown in Figure 1(e), minimizing this energy instead of the Euclidean one yields a significantly better result at the cost of having to compute geodesic distances. Motivated by this idea, we propose to use this energy as the main building block in a shape mapping algorithm. We reformulate it to allow efficient optimization and combination with other terms that address the case of positively curved target surfaces $M_{2}$, as described in the following section.

\section{REVERSIBLE HARMONIC MAPS}

Notation. We represent a triangle mesh $M$ by its vertex, edge, and face sets $(\mathcal{V}, \mathcal{E}, \mathcal{F})$, respectively, where we denote $n=|\mathcal{V}|$, and its given embedding by $V \in \mathbb{R}^{n \times 3}$. We denote scalar functions $g: M \rightarrow \mathbb{R}$ by a vector of coefficients of piecewise linear hat functions, with $g \in \mathbb{R}^{n}$. The squared $l_{2}$ norm of a function on the surface is given by $\|g\|_{M}^{2}=g^{T} A g$, where $A \in \mathbb{R}^{n \times n}$ is the diagonal (lumped) mass matrix of the vertices. The total area of the mesh is denoted by $s=\operatorname{tr}(A)$. The squared gradient norm is given by $\|g\|_{W}^{2}=g^{T} W g$, where $W$ is the matrix of cotangent weights. Similarly, for matrices $G \in \mathbb{R}^{n \times k}$ whose columns are scalar functions, we use the matrix trace: $\|G\|_{M}^{2}=\operatorname{tr}\left(G^{T} A G\right),\|G\|_{W}^{2}=\operatorname{tr}\left(G^{T} W G\right)$. When two meshes are involved, we use a subscript; e.g., $A_{i}$ is the mass matrix of $M_{i}$.

\subsection{Energy}

While the geodesic harmonic energy can be effective, harmonic maps in general can become degenerate and map large regions to a single point. Indeed, the map taking all the points on $M_{1}$ to a single point on $M_{2}$ is harmonic. An example of such behavior is demonstrated in Figure 2. On the top row, the source $M_{1}$ is a sphere with a small number of triangles (a) that is mapped to a high-resolution target sphere $M_{2}$. Even if the initial map is the ground-truth map (b) between the spheres, during the optimization the map quickly "slides over" to a single hemisphere of the target sphere (c) and then degenerates and collapses to a single point (d). The same phenomenon occurs for complex shapes, as shown in the bottom row. Here, we initialized a map between two bird shapes from SHREC'07 (Giorgi et al. 2007) (e,f) using landmarks as specified in Section 4.2. Initially (f), the tips of the wings and tail are mapped correctly, but again they gradually slide (g) until no vertices are mapped to most of the target's wings or tail (h).

Intuitively, in the discrete case, we can think of $M_{1}$ as an elastic fishnet instead of a continuous rubber sheet, stretched over $M_{2}$ and allowed to compress. Then, in addition to the usual degeneracies in the smooth case, the target surface can effectively "slip through" one of the holes in the net, allowing the map to degenerate to a

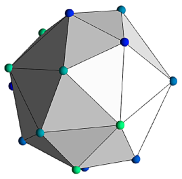

(a)

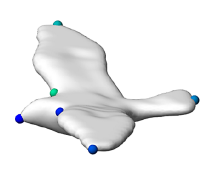

(e)

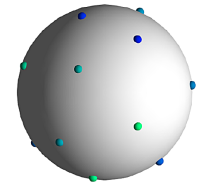

(b)

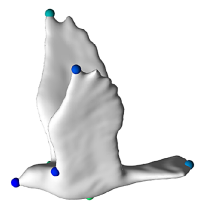

(f)

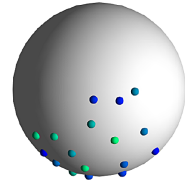

(c)

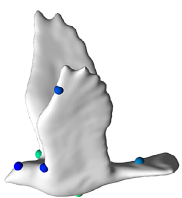

(g)

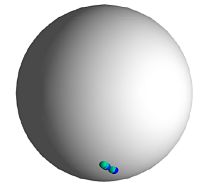

(d)

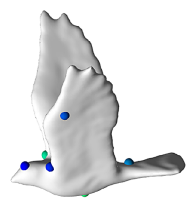

(h)
Fig. 2. Collapse of a harmonic map. Top row: mapping from a lowresolution sphere (a) to a high-resolution sphere, starting from the identity map (b). The map quickly "slides" to a single hemisphere (c) and then degenerates (d). Bottom row: the same phenomenon with more complex shapes from SHREC'07 (Giorgi et al. 2007), where we use a sparse set of landmarks for initialization and visualization. The final result (h) does not map any points to the upper part of the wings and to most of the tail.

single point. To prevent this, we minimize the geodesic harmonic energies of both the forward and backward maps, together with a reversibility constraint relating both maps. As we later demonstrate, this approach is highly effective in generating nondegenerate harmonic maps.

Smoothness. Given two triangle meshes $M_{1}, M_{2}$ and maps $\phi_{12}, \phi_{21}$, the total harmonic energy of both forward and backward maps is given by

$$
E_{D}\left[\phi_{12}, \phi_{21}\right]=\sum_{\substack{i, j \in\{1,2\} \\ i \neq j}} \frac{1}{s_{j}} E_{D}\left[\phi_{i j}\right]
$$

where $E_{D}$ is given in Equation (4).

Reversibility. We define the reversibility energy similarly to Ezuz and Ben-Chen (2017) and Kovnatsky et al. (2013) as

$$
E_{R}\left[\phi_{12}, \phi_{21}\right]=\sum_{\substack{i, j \in\{1,2\} \\ i \neq j}} \frac{1}{s_{i}^{2}} \sum_{p_{i} \in \mathcal{V}_{i}} d_{M_{i}}^{2}\left(\phi_{j i}\left(\phi_{i j}\left(p_{i}\right)\right), p_{i}\right) A_{i}\left(p_{i}\right) .
$$

The reversibility energy prevents the maps from collapsing. In the smooth case, if the reversibility energy is bounded pointwise, it easily follows that the maps are close to being injective and surjective, as we show in the appendix. For both energies, care is required to handle correctly meshes of different scales, hence the normalization by $s_{i}$, the total area of $M_{i}$.

Finally, the full energy is given by

$$
E\left[\phi_{12}, \phi_{21}\right]=\alpha E_{D}\left[\phi_{12}, \phi_{21}\right]+(1-\alpha) E_{R}\left[\phi_{12}, \phi_{21}\right],
$$

where the parameter $\alpha \in[0,1]$ controls the tradeoff between smoothness and reversibility. Note that while in the continuous setting exact reversibility might be desired, this will not be the case in the discrete setting described in Section 3.2. Thus, we enforce reversibility as a soft constraint controlled by the parameter $\alpha$. 


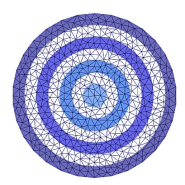

Target Texture
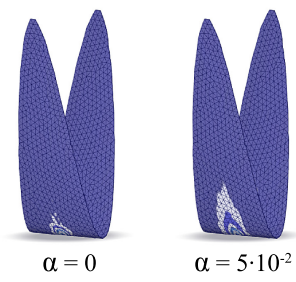

$\alpha=5 \cdot 10^{-2}$

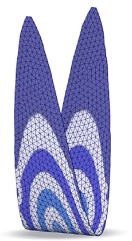

$\alpha=5 \cdot 10^{-4}$

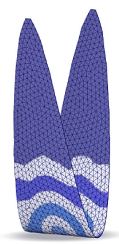

$\alpha=5 \cdot 10^{-6}$

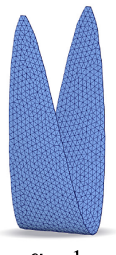

$\alpha=1$
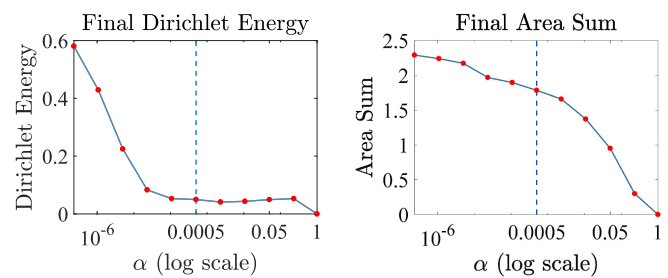

Fig. 3. Preventing collapse with reversibility. For different $\alpha$ values, we measure the discrete geodesic Dirichlet energy and the sum of the relative mapped area (ideally 2). We visualize some of the results using texture transfer (left) and show the final values as a function of $\alpha$ (right). Note that when $\alpha$ is small, the Dirichlet energy is high, and when $\alpha$ is large, the map collapses, as is evident by the zero total area. Finally, taking $\alpha=5 \cdot 10^{-4}$ leads to a good balance between the energy components.

To demonstrate the effect of the different components of the energy, we compute a map between a disk and a folded disk using different values of $\alpha$. In the initial map, all the interior vertices of the disk are mapped to a single interior vertex of the folded disk, and the boundary of the source is mapped to the boundary of the target. During the optimization, the mapped boundary vertices are not constrained to lie on the boundary of the target.

Figure 3 shows the results, where we visualize the maps using texture transfer (left) and quantitatively evaluate them using the discrete geodesic Dirichlet energy from Equation (4) (center right) as well as the sum of the total area of the images of the forward and backward maps (right). The graphs show the final values of these quantities as a function of the parameter $\alpha$, where the energy is normalized with respect to its value in the first iteration.

The figure demonstrates the tradeoff that the parameter $\alpha$ controls; e.g., taking $\alpha=0$ models reversibility only, leading to a high Dirichlet energy. On the other hand, taking $\alpha=1$ models harmonicity only and leads to a map that collapses the image to a single point, as is evidenced by the final total area, which is zero. In general, if $\alpha$ is too small, the map has a high Dirichlet energy and thus is more distorted locally, and if $\alpha$ is too large, the map collapses. Taking $\alpha=5 \cdot 10^{-4}$, as we did for all the experiments except for Figure 3, leads to a balance between the harmonic energy and reversibility.

Minimizing the energy in Equation (7) requires computing the gradient of the geodesic distances with respect to the forward and backward maps, as well as tracing vector fields on the surface, which are both computationally heavy. We therefore apply two approximations to address these issues.

\subsection{Energy Approximation}

3.2.1 Notation. Any point $p \in M$ can be represented uniquely using its barycentric coordinates $\omega_{l}(p), l \in\{1, . ., 3\}$ with respect to the face $f(p)=\left(v_{1}, v_{2}, v_{3}\right) \in \mathcal{F}$ it lies on. We denote by $\lambda(p) \in \mathbb{R}^{1 \times n}$ the row vector that is zero everywhere except at the vertices of $f(p)$, where we have $\lambda(p)\left[v_{l}\right]=\omega_{l}(p)$. In addition, we denote the feasible row set of $M$, i.e., the set of all possible such vectors, by $\mathcal{P}=$ $\{\lambda(p) \mid p \in M\}$. Finally, the feasible set of all possible precise maps from $M_{1}$ to $M_{2}$ is given by $\mathcal{P}_{12}=\left\{P_{12} \in \mathbb{R}^{n_{1} \times n_{2}} \mid P_{12}(l,:) \in \mathcal{P}_{2}, \quad \forall l \in\right.$ $\left.\left\{1 . . n_{1}\right\}\right\}$, where $P(l,:)$ denotes the $l$ th row of the matrix $P$. Thus, any map $\phi_{12}$ can be represented using a matrix $P_{12}$ by setting $P_{12}(l,:)=$ $\lambda_{2}\left(\phi_{12}\left(v_{l}\right)\right), \forall l \in\left\{1 . . n_{1}\right\}$, which, by definition, is in the feasible set $\mathcal{P}_{12}$. Furthermore, the matrix $P_{12} V_{2} \in \mathbb{R}^{n_{1} \times 3}$ represents the images of the vertices $\mathcal{V}_{1}$ under the map $\phi_{12}$.
3.2.2 High-Dimensional Embedding. As we have seen, if the target space is Euclidean, then the geodesic distances are Euclidean distances, and the optimization is simple and efficient. Following similar ideas in the literature (Bronstein et al. 2006), we therefore suggest to use a high-dimensional Euclidean embedding as a proxy for fast geodesic distance computation.

Given a mesh $M$, we seek an embedding $x: \mathcal{V} \rightarrow \mathbb{R}^{m}$ for $m \ll$ $n$ such that the Euclidean distance $\|x(u)-x(v)\|$ approximates well the geodesic distance $d_{M}(u, v)$ for all $u, v \in \mathcal{V}$. The literature on such embeddings is quite vast, and we chose to leverage the method suggested by Panozzo et al. (2013) that relies on multidimensional scaling (Cox and Cox 2000). Any other embedding method could be used as well, as long as the geodesic distances are well approximated. We took $m=8$ in all our experiments, following Panozzo et al. (2013).

Our goal now is to compute a harmonic map between the high-dimensional Euclidean embeddings. We denote by $X \in \mathbb{R}^{n \times m}$ the matrix whose rows are the embeddings $x(v), \forall v \in \mathcal{V}$. Rewriting the harmonic and reversibility energies in terms of the highdimensional embeddings, and in matrix form, leads to

$$
E\left(P_{12}, P_{21}\right)=\sum_{\substack{i, j \in 1,2\} \\ i \neq j}} \alpha \frac{1}{s_{j}}\left\|P_{i j} X_{j}\right\|_{W_{i}}^{2}+(1-\alpha) \frac{1}{s_{i}^{2}}\left\|P_{i j} P_{j i} X_{i}-X_{i}\right\|_{M_{i}}^{2} .
$$

Note that the weights in the harmonic energy, given now in matrix form in $W_{i}$, remain the same for the high-dimensional embedding, since the embedding is nearly isometric.

Figure 4 demonstrates the importance of the high-dimensional embedding. For shapes where the geodesic distances are considerably different than Euclidean distances in $\mathbb{R}^{3}$, e.g., the spring shapes from SHREC'07 (Giorgi et al. 2007), using the threedimensional input Euclidean embedding in the optimization leads to a highly distorted map (center). Specifically, neighboring vertices on the source shape are mapped to different coils of the target spring, which are extrinsically close but intrinsically far. On the other hand, by using the high-dimensional embedding, the geodesic Dirichlet energy is well approximated, leading to an improved map (right).

3.2.3 Half-Quadratic Splitting. While Equation (8) can be minimized using gradient descent, we found, similarly to Ezuz and Ben-Chen (2017), that it is more efficient to use the half-quadratic splitting optimization method (Geman and Yang 1995) (see also, e.g., Wang et al. (2008) and Zoran and Weiss (2011)). We introduce 


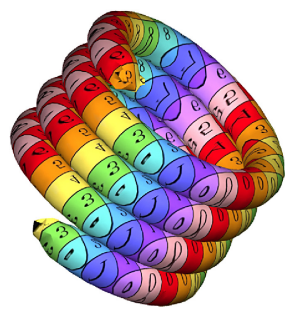

Target Texture
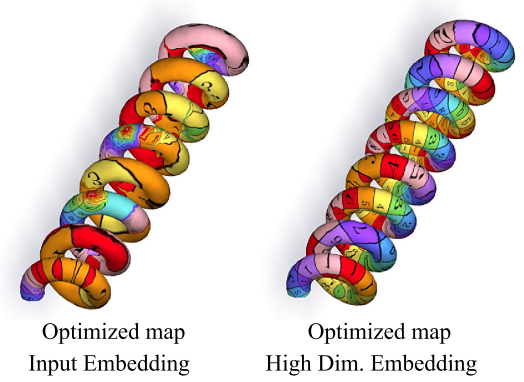

Fig. 4. The importance of the high-dimensional embedding. For these shapes, geodesic and Euclidean distances are significantly different; thus, using the input vertex positions in $\mathbb{R}^{3}$ during the optimization results in a highly distorted map (center). The high-dimensional embedding that approximates the geodesic distances leads to a better map (right).

auxiliary variables $X_{i j} \in \mathbb{R}^{n_{i} \times m}$, which estimate the images of the vertices $\mathcal{V}_{i}$ given by $P_{i j} X_{j}$. Substituting, the energies are

$$
\bar{E}_{D}\left(X_{i j}\right)=\frac{1}{s_{j}}\left\|X_{i j}\right\|_{W_{i}}^{2}, \quad \bar{E}_{R}\left(P_{i j}, X_{j i}\right)=\frac{1}{s_{i}^{2}}\left\|P_{i j} X_{j i}-X_{i}\right\|_{M_{i}}^{2},
$$

for $i, j \in\{1,2\}, i \neq j$. In addition, we need soft constraints for the auxiliary variables:

$$
\bar{E}_{Q}\left(P_{i j}, X_{i j}\right)=\frac{1}{s_{i} s_{j}}\left\|X_{i j}-P_{i j} X_{j}\right\|_{M_{i}}^{2},
$$

where we again normalize by $s_{i} s_{j}$ to retain scale invariance.

The full energy is now

$$
\begin{aligned}
& \bar{E}\left(P_{12}, P_{21}, X_{12}, X_{21}\right)= \\
& \sum_{\substack{i, j \in 1,2\} \\
i \neq j}} \alpha \bar{E}_{D}\left(X_{i j}\right)+(1-\alpha) \bar{E}_{R}\left(P_{i j}, X_{j i}\right)+\beta \bar{E}_{Q}\left(P_{i j}, X_{i j}\right),
\end{aligned}
$$

where $\beta$ controls the accuracy of the auxiliary variables and functions as a step size. When using the half-quadratic splitting optimization scheme, the update schedule for $\beta$ is often tailored per application, with the general guideline of increasing $\beta$ as the iterations advance (Wang et al. 2008). In our case, we often initialize the optimization with a highly degenerate map, e.g., as obtained from a sparse set of landmarks, and therefore the value of $\beta$ during the first iterations should be small enough so that the map can change significantly. As the iterative solution approaches a local optimum, $\beta$ can increase, as less modification is required. The final value of $\beta$ should be large enough to ensure $P_{i j}$ and $X_{i j}$ correspond. In all of our experiments, we took $\beta=5 \cdot 10^{-3} k$, where $k$ is the optimization iteration number for the first 100 iterations, and then kept the value of $\beta$ fixed until convergence.

\subsection{The Optimization Problem}

Our optimization problem is now given by

$$
\begin{array}{cl}
\underset{P_{12}, P_{21}, X_{12}, X_{21}}{\operatorname{minimize}} & \bar{E}\left(P_{12}, P_{21}, X_{12}, X_{21}\right) \\
\text { subject to } & P_{12} \in \mathcal{P}_{12}, P_{21} \in \mathcal{P}_{21},
\end{array}
$$

where $\mathcal{P}_{i j}$ is the feasible set of precise maps from $M_{i}$ to $M_{j}$. Despite the two approximations that we used, solving this optimization problem succeeds in decreasing the total discrete Dirichlet energy from Equation (4) while preventing the map from collapsing, as illustrated in Figure 5. In addition to the energy, we show the initial map that was created from landmarks as described in Section 4.2 and the intermediate map at a few iterations.

\section{OPTIMIZATION}

Our optimization problem has a block structure, in the sense that if some of the variables are kept fixed, it becomes a linear least squares problem. We therefore chose to use block coordinate descent (see, e.g., Xu and Yin (2013)) as the optimization algorithm.

\subsection{Block Coordinate Descent}

In each subiteration, we solve for one of the matrices $X_{i j}, P_{i j}$ while keeping the others fixed. Since the energy is quadratic in all the variables, every subiteration involves a relatively simple optimization problem, with the only complication arising because of the nonconvex feasible sets $\mathcal{P}_{i j}$.

Optimizing for $X_{i j}$. When $P_{12}, P_{21}$ are fixed, the optimization problem is a linear least squares minimization of the form $\| A X_{i j}-$ $B \|_{2}^{2}$, with known matrices $A$ and $B$, where $A$ is sparse, which we solve using a direct method. The system is highly overconstrained, as even if $P_{j i}$ degenerates, the system is well conditioned due to the term $\bar{E}_{Q}$, as long as the vertex areas of the mesh $M_{i}$ do not vanish.

Optimizing for $P_{i j}$. When $X_{12}, X_{21}$ are fixed, the energy has the form $\left\|P_{i j} A-B\right\|_{2}^{2}$, where $A, B$ are known, with the constraints that $P_{i j} \in \mathcal{P}_{i j}$. Following Ezuz and Ben-Chen (2017), the optimization is done by solving for every row of $P_{i j}$ separately. Intuitively, we can think of $B$ as a high-dimensional embedding of the faces of $M_{j}$, and of $A$ as a high-dimensional point cloud. The optimal $P_{i j}$ projects each point in $A$ to its closest point on the faces given by $B$. As shown in Ezuz and Ben-Chen (2017), this process is guaranteed to find $P_{i j}$ that are globally optimal when $X_{i j}$ are kept fixed.

Stopping Criterion. The alternating descent guarantees that the energy is reduced at every iteration, since the subiterations find a global optimum of the reduced optimization problems. In practice, we stopped the optimization when the change of energy was less than $10^{-9}$, or after a maximum of $N=200$ iterations. In most cases, we have observed convergence of the energy to high precision even when early stopping after $N$ iterations were used.

We provide the details of the alternating descent in Algorithm 1.

\begin{tabular}{l}
\hline ALGORITHM 1: Alternating minimization \\
\hline \hline Input: Two triangle meshes $M_{1}, M_{2}$, initial $P_{12}, P_{21}, X_{12}, X_{21}$ \\
Output: $P_{12}, P_{21}$ \\
For $k=1 \ldots N$ \\
$\quad$ For $i=1,2$ \\
$\quad j=3-i$ \\
$\quad P_{i j} \leftarrow \underset{P \in \mathcal{P}_{i j}}{\operatorname{argmin}} \bar{E}_{R}\left(P, X_{j i}\right)+\bar{E}_{Q}\left(P, X_{i j}\right)$ \\
$\quad X_{i j} \leftarrow \underset{X \in \mathbb{R}^{n_{i} \times m}}{\operatorname{argmin}} \bar{E}_{D}(X)+\bar{E}_{R}\left(P_{j i}, X\right)+\bar{E}_{Q}\left(P_{i j}, X\right)$ \\
end \\
end
\end{tabular}




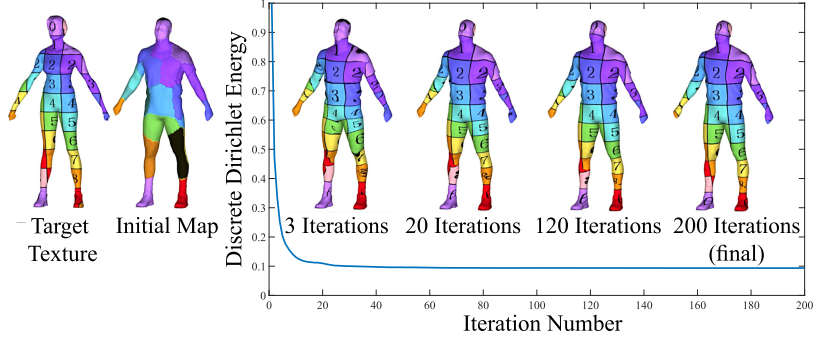

Fig. 5. Optimization of Equation (12), starting from landmarks. The discrete Dirichlet energy in Equation (4) decreases, and the final map, visualized by texture transfer, is semantic and not distorted locally.

\subsection{Initialization}

Our method is general and can receive as input various initial data. Depending on the input, we describe the initialization of the variables $P_{i j}, X_{i j}$. Given a pointwise map $\phi_{12}: \mathcal{V}_{1} \rightarrow M_{2}$, its corresponding matrix representation is given by $P_{12}(l,:)=\lambda_{2}\left(\phi_{12}\left(v_{l}\right)\right)$ for $l \in\left\{1, . ., n_{1}\right\}$. Similarly, given a matrix representation $P_{i j} \in \mathcal{P}_{i j}$, we have that $\phi_{i j}\left(v_{l}\right)=\left(P_{i j} V_{j}\right)(l,:) \in M_{j}$. Therefore, in the following, refer to $P_{i j}$ or $\phi_{i j}$ according to which notation is more convenient.

Pointwise Map. Given a pointwise map $P_{12}$, we approximate an inverse map $P_{21}$ by taking $\phi_{21}\left(v_{2}\right)=\operatorname{argmin}_{v \in V_{1}}\left\|\phi_{12}(v)-v_{2}\right\|$. Then, the initialization of $X_{i j}$ is $P_{i j} X_{j}$.

Functional Map. The term functional map (Ovsjanikov et al. 2012) denotes a map between scalar functions. It is a linear operator that can be represented using a matrix when scalar functions are represented in a linear basis. Let $\Psi_{i} \in \mathbb{R}^{n_{i} \times k_{i}}$ be a matrix whose columns are basis functions of a subspace of scalar functions on $M_{i}$, where each function is piecewise linear and is defined by values assigned to vertices. Given a pointwise map that maps vertices of $M_{1}$ to points on $M_{2}$, the corresponding functional map $C_{12} \in \mathbb{R}^{k_{1} \times k_{2}}$ maps functions on $M_{2}$ to functions on $M_{1}$, represented in the reduced basis. Therefore, given two functional maps $C_{12}$ and $C_{21}$, we initialize $X_{i j}=\Psi_{i} C_{i j} \Psi_{j}^{\dagger} X_{j}$. Optimizing $P_{i j}$ does not require initialization. Figure 9 shows results where functional maps were used for initialization.

Landmarks. Given $r$ input landmark pairs $\left\{\left(p_{i}, q_{i}\right)\right\}$ where $p_{i} \in \mathcal{V}_{1}, q_{i} \in \mathcal{V}_{2}$ and $i \in\{1, \ldots, r\}$, we first construct a rough initial pointwise map $P_{12}$ and then use it to initialize the rest of the variables, as previously described. We first compute the geodesic Voronoi diagram on $M_{1}$ with centers $p_{i}$, and then set $\phi_{12}(v)=q_{i}, \forall v \in C_{i}$, where $C_{i}$ is the geodesic cell corresponding to the center $p_{i}$. Note that this initialization is highly degenerate, as all the points on $M_{1}$ are mapped to the landmarks $q_{i}$ on $M_{2}$, yet it is enough for our needs. In Figure 5, initialization using input landmarks is visualized.

\subsection{Limitations}

Using the projection step when optimizing for $P_{i j}$ has some limitations. First, as discussed in Panozzo et al. (2013), such a projection is not smooth. Furthermore, the closest point on the high-dimensional embedding of the triangle mesh might not be unique; therefore the solution of the optimization for $P_{i j}$ might alternate between two configurations with the same energy. Thus, while the energy is guaranteed to converge, we do not have a similar guarantee for the convergence of the solution. In practice, we have not encountered a case where these limitations posed a practical problem. In future work, it could be possible to handle the first issue using a Phong projection, as in Panozzo et al. (2013), and the second issue using an additional regularization that penalizes diverting from the current solution.

Since we optimize for both harmonicity and reversibility, we cannot guarantee convergence to a smooth harmonic map; this is likely a fruitful av-
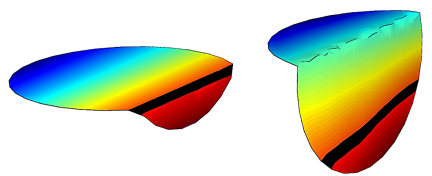
enue for future work. In addition, our method does not consider extrinsic features such as edges and corners, and hence such features will not necessarily be mapped to each other. An example is shown in the inlined figure, where our map between the two folded disks is smooth, but the edge features do not correspond.

\subsection{Timing}

The most expensive step in the optimization process is the projection on a triangle mesh for optimizing $P_{i j}$. However, this procedure is highly parallelizable since the projection of each point is independent of the other points. We used CUDA 8 to implement the projection in parallel, while the rest of the optimization method was written in MATLAB. On a desktop machine with a TITANX GPU and an Intel Core i7 processor, 200 optimization iterations of our method, for shapes with $5 \mathrm{~K}$ vertices, took around 115 seconds.

\section{RESULTS}

To validate our method, we have compared with a variety of stateof-the-art mapping techniques, in accordance with the type of input they can accept. In addition, we show applications to shape interpolation and quad mesh transfer.

\subsection{Quality Metrics}

To evaluate the quality of a map, we measure its smoothness through its conformal distortion and its semantic accuracy, using the distance to the ground truth, when given. We also use alternative measures, such as symmetry and compatibility with ground-truth segmentations, when no dense ground-truth map is available.

Conformal Distortion. We use the definition by Hormann and Greiner (2000, Equation (3)) for the conformal distortion of a single triangle $f \in \mathcal{F}_{1}: \kappa(f)=\frac{\sigma_{1}}{\sigma_{2}}+\frac{\sigma_{2}}{\sigma_{1}}$, where $\sigma_{1} \geq \sigma_{2}$ are the singular values of the linear transformation that maps $f$ from $M_{1}$ to $M_{2}$. We subtract 2 so that the minimal conformal distortion is zero and visualize the result as a cumulative graph showing the percentage of triangles with less than a certain distortion value.

Distance from Ground Truth. When a ground-truth map is given, we measure the distance from the ground truth using the protocol suggested by Kim et al. (2011, Section 8.2). For every mapped vertex, we measure its geodesic distance from the ground-truth 

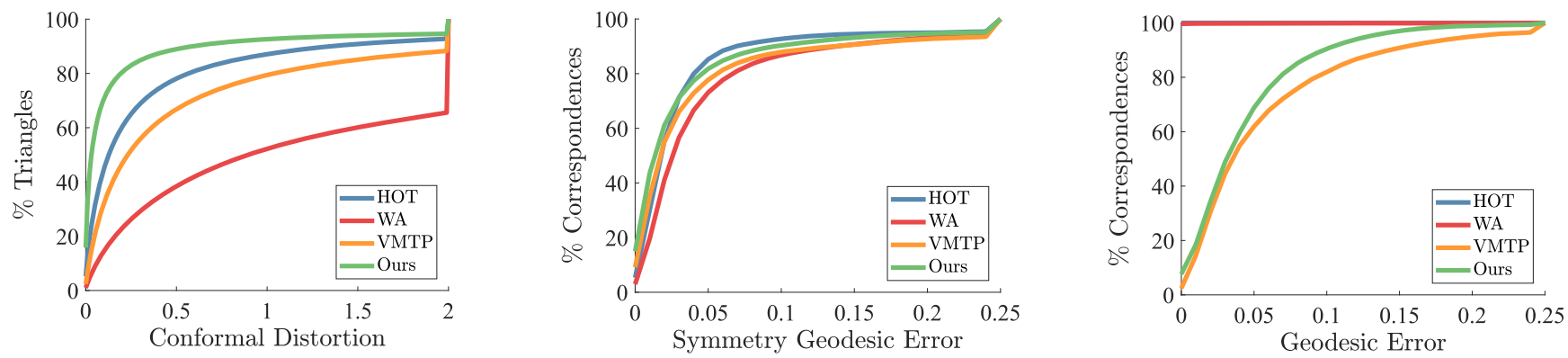

Fig. 6. Quantitative comparison on the SHREC dataset, measuring, from left to right, conformal distortion, compatibility with symmetries, and distance from ground-truth landmarks. Note that we achieve a better conformal distortion and comparable symmetry geodesic error. Furthermore, note that WA and HOT do not modify their input landmarks, while our method and VMTP do. Compared to VMTP, we achieve a better landmark geodesic error.

location, relative to the square root of the total area of $M_{2}$, and visualize the percent of vertices whose distortion is less than a given value.

Compatibility with Segmentations. For some datasets groundtruth labeled segmentations are available. In this case, for every pair of shapes and a given map we measure the consistency of the segmentation with respect to the map. This is done by computing the relative vertex area of vertices that are mapped to a face that belongs to the same segment as the source vertex.

Compatibility with Symmetry. For some datasets a ground-truth map is only known for a subset of the points, yet a full intrinsic symmetry can be computed for every shape separately. We assume that a good map should respect the intrinsic symmetries of the source and target shapes, given by $S_{1}, S_{2}$, respectively. We therefore use these symmetries as input and measure the compatibility of the map $\phi_{12}$ with the symmetries, given by the geodesic distance $d_{M_{2}}\left(S_{2}\left(\phi_{12}\left(v_{1}\right)\right), \phi_{12}\left(S_{1}\left(v_{1}\right)\right)\right), \forall v_{1} \in \mathcal{V}_{1}$. We visualize the result using a cumulative graph, similarly to the ground-truth error. To compute the symmetries, we use the method by Kim et al. (2011). We also manually filtered the results to use only the accurate symmetries.

\subsection{Dataset: SHREC, Input: Landmarks}

We use the BIM benchmark (Kim et al. 2011) that provides more than 200 pairs of highly nonisometric shapes from the SHREC dataset (Giorgi et al. 2007) with user-verified landmarks. We compare our method with a state-of-the-art parameterization-based method (Aigerman and Lipman 2016) (HOT) and the weighted averages method (Panozzo et al. 2013) (WA). Both receive as input landmark points, which are not modified during the optimization, and generate precise maps. In addition, we compare to the recent method by Mandad et al. (2017) (VMTP), which similarly gets as input landmark points yet can modify them during the optimization. Since VMTP requires uniform isotropic meshes, we recursively add edges using the longest edge bisection method to meshes with fewer than $10 \mathrm{~K}$ vertices before applying VMTP. All the methods we compare with produce precise maps, as vertex-to-vertex maps induce high local distortion. As input to our method we also use the user-defined landmarks and extend them to a full initial map as described in Section 4.2. The landmarks are not used after the initialization.

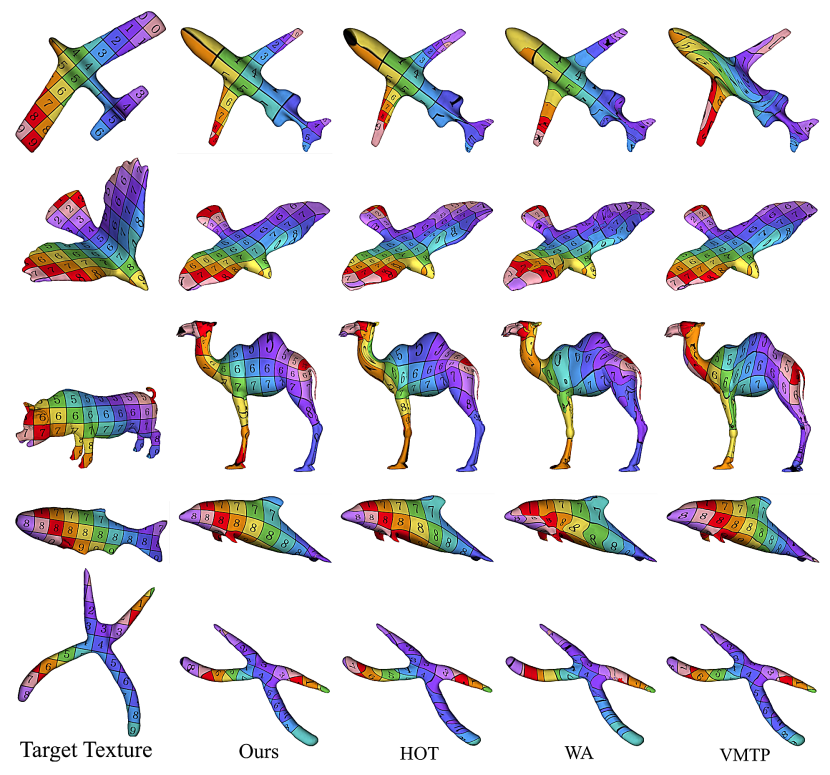

Fig. 7. Qualitative results, from input landmarks. From left to right: target texture, our method, HOT (Aigerman and Lipman 2016), WA (Panozzo et al. 2013), and VMTP (Mandad et al. 2017). See the text for details.

Quantitative results are shown in Figure 6, where we measure conformal distortion, compatibility with symmetries, and distance from the ground-truth landmarks. Note that our method achieves the best conformal distortion. In addition, the distance from the ground-truth landmarks is also improved when compared to the other method that modifies them (VMTP). As shown in Section 5.3, the option to modify the input landmarks is valuable when the input is not completely reliable. In terms of compatibility with symmetry, our method is comparable with existing techniques, notably achieving a better ratio of perfect matches with about $15 \%$ of the vertices exactly symmetric for our method, where the next best method has less than $10 \%$ exactly symmetric vertices. On this dataset, ground-truth segmentations are also available (Chen et al. 2009; Kalogerakis et al. 2010), and measuring the relative mapped area that is compatible with the segmentations, we have HOT: $90.39 \%$, WA: $90.35 \%$, VMTP: $81.8 \%$, and our method: $89.62 \%$. Therefore, this measure also demonstrates that our maps are as 

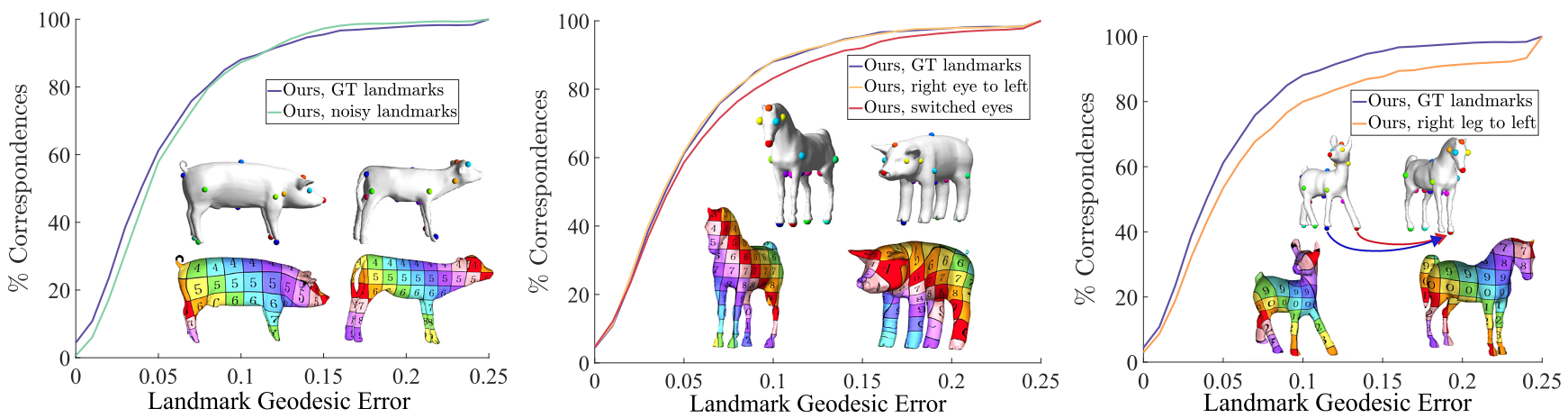

Fig. 8. Mapping SHREC quadrupeds by our method, starting from noisy landmarks. Our method is only slightly affected by the noise even when the landmark modification is severe.

compatible semantically as existing techniques while being considerably more conformal.

Qualitative results are shown in Figure 7, where we have selected a subset of pairs to visually show the differences between the maps. In every row, we show, from left to right, the target texture, and the results of our method, HOT, WA, and VMTP.

\subsection{Dataset: SHREC Quadrupeds, Input: Noisy Landmarks}

In many cases, the selection of the landmarks by the user has some variability (see, e.g., Chen et al. (2012)), and it might be better to treat these landmarks as guidelines rather than exact ground truth. Our approach is compatible with this notion, since our method only uses the landmarks for initialization, and their final location will, in most cases, vary from their initial one. To check the sensitivity of our approach to the landmark locations, we repeated the experiment from Section 5.2 with various landmark modifications. Figure 8 shows the landmark geodesic error of the output maps when starting from the noisy landmarks compared to starting from the original landmarks. We ran the experiment on the "quadrupeds" class (20 pairs) from the SHREC dataset and did the following modifications: (1) moved every landmark randomly to a vertex in its five-ring neighborhood, (2) switched between the two eyes or mapped both eyes on $M_{1}$ to a single eye on $M_{2}$, and (3) mapped both feet on $M_{1}$ to the same foot on $M_{2}$. In addition to the error graph, we show some example maps, as well as the input noisy landmarks. As the figure shows, our results are not sensitive to landmark noise, and even a relatively severe modification, such as mapping both feet to the same foot, yields good qualitative and quantitative results.

\subsection{Dataset: SHREC Two Pairs, Input: Functional Map}

The functional map (Ovsjanikov et al. 2012) machinery is quite versatile and allows one to compute generalized maps in a variety of cases. Our method can also be used to extract a precise pointwise map from a given functional map. We use the SHREC dataset with its landmark data from the BIM benchmark (Kim et al. 2011) and use the landmarks to compute a functional map using the Wave Kernel Map and the Wave Kernel Signature (Aubry et al. 2011). Any other recent method for computing functional maps could be used as well. We provide the functional map as input to our approach
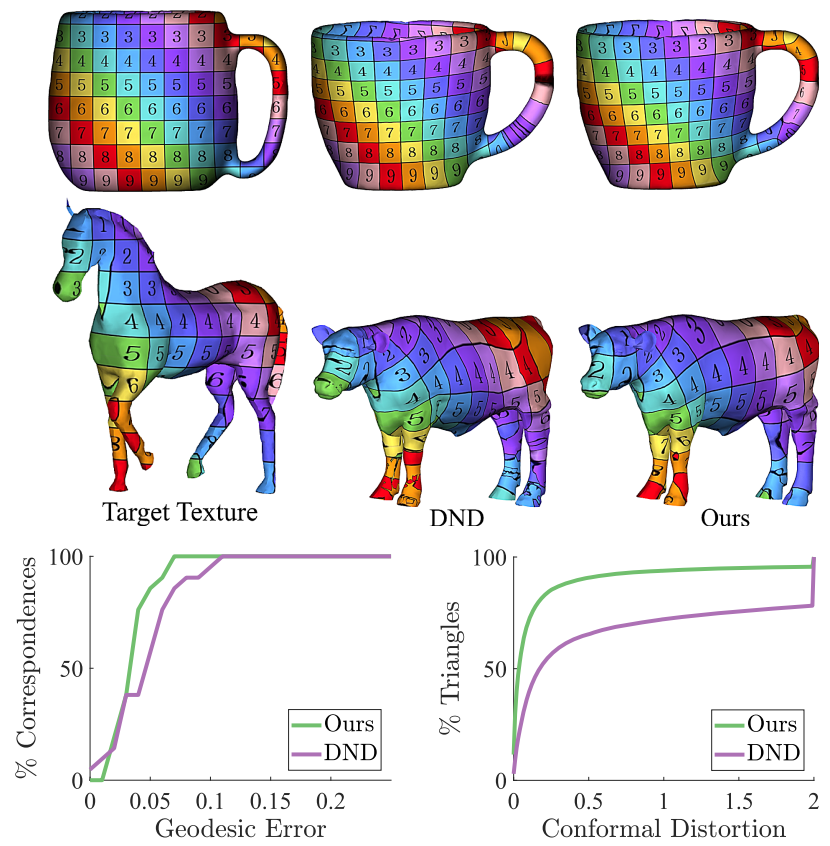

Fig. 9. Qualitative and quantitative comparison starting with a functional map computed from landmarks. From left to right: target texture, Ezuz and Ben-Chen (2017) (DND), our method. Notice the difference at the cup handle and the legs.

and the recent map deblurring approach (Ezuz and Ben-Chen 2017) (DND), which is the only other method that recovers precise maps from a functional map. Specifically, we used the consistency extension of DND with $\alpha=0.8$. Figure 9 qualitatively visualizes the difference between the methods for two pairs of shapes. Note the map improvement on the handle of the cup and the legs of the cow. We also show graphs of the conformal distortion and ground-truth error of the landmarks.

\subsection{Dataset: Caricatures, Input: Landmarks}

One of the advantages of our formulation is its simplicity, which leads to flexibility in adding additional components to the energy. For example, in some cases, it can be beneficial to add weak 

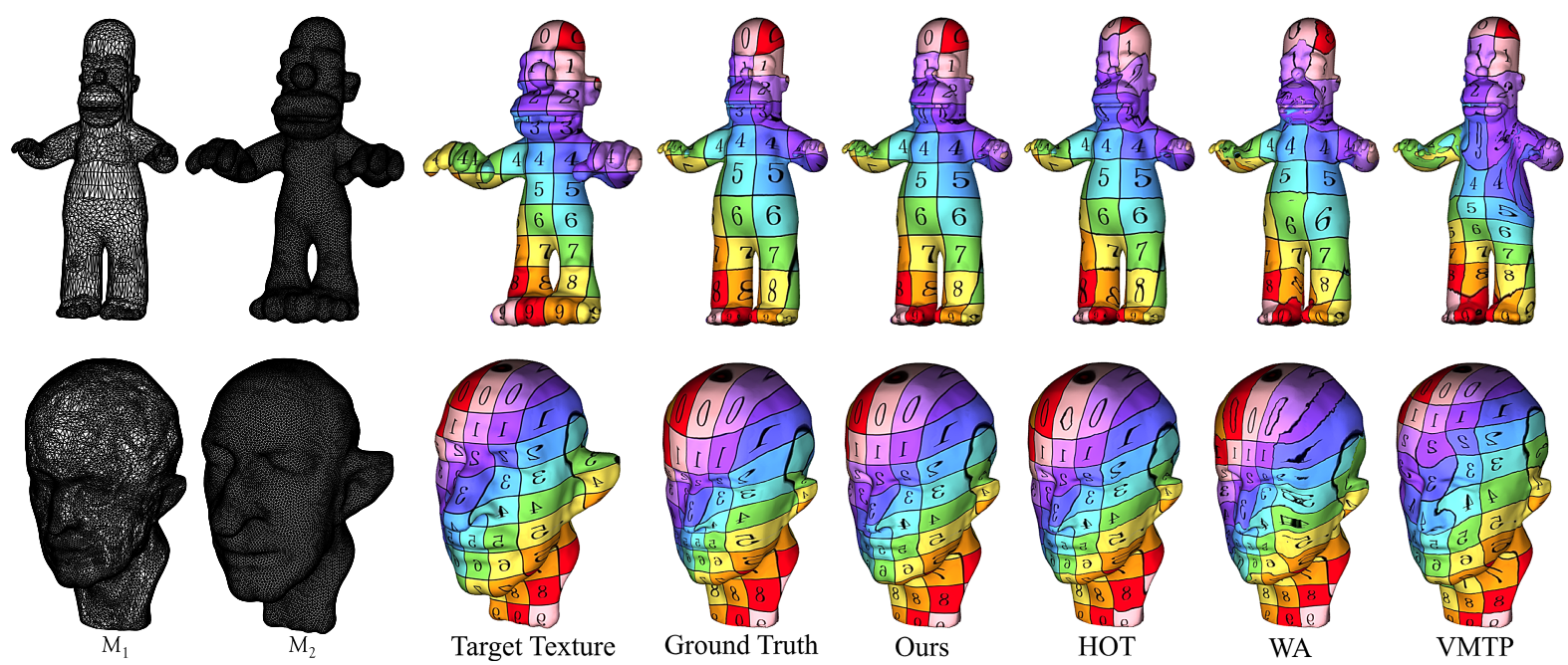

Fig. 10. Qualitative result, caricatures. $M_{2}$ was generated by deforming $M_{1}$ (Sela et al. 2015), and the deformation defines a ground-truth map. $M_{2}$ was remeshed so that the source and target shapes do not have the same connectivity.
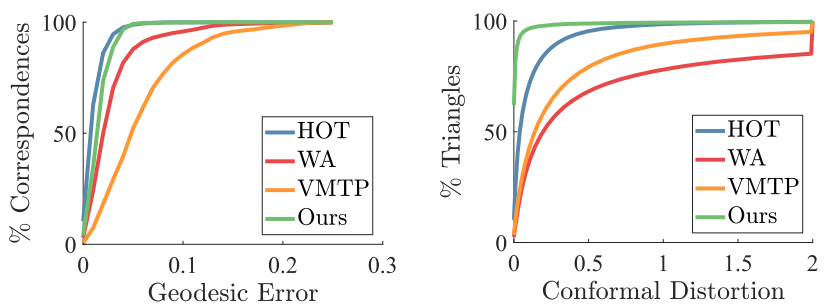

Fig. 11. Quantitative measures for the meshes in Figure 10. Note that our method achieves a considerably better conformal distortion while maintaining ground-truth error comparable to existing methods.

landmark constraints to encourage feature points to remain in the neighborhood of the input landmarks.

Weak Landmark Constraints. Given pairs of matching landmarks $p_{i} \in \mathcal{V}_{1}, q_{i} \in \mathcal{V}_{2}, i=1 \ldots r$, we add the following term to the energy:

$\gamma \sum_{i=1}^{r} A_{1}\left(p_{i}\right)\left\|X_{12}\left(p_{i},:\right)-X_{2}\left(q_{i}\right)\right\|_{M_{2}}^{2}+A_{2}\left(q_{i}\right)\left\|X_{21}\left(q_{i},:\right)-X_{1}\left(p_{i}\right)\right\|_{M_{1}}^{2}$

The value of $\gamma$ depends on the reliability of the landmarks; if the landmarks are not accurate, then $\gamma$ should be small. To demonstrate the effectiveness of this approach, we used as input the Homer and Max Planck models, and caricatures of these models generated using the method by Sela et al. (2015). The caricatures have the same triangulation as the original shapes. We remesh the caricatures to avoid bias and project the original caricature to the remeshed model to generate the ground truth for validation. As input to all approaches, we picked 28 and 14 landmarks from the input map for the Homer and Max models, respectively. For our algorithm we added the energy in Equation (13) with $\gamma=1$. The qualitative and quantitative results are shown in Figures 10 and 11, respectively. As in previous experiments, we achieve considerably better conformal distortion, with comparable ground-truth error.

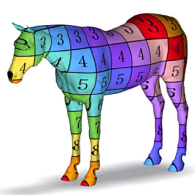

Target Texture

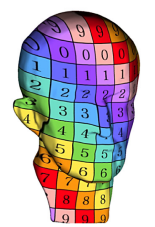

Target Texture
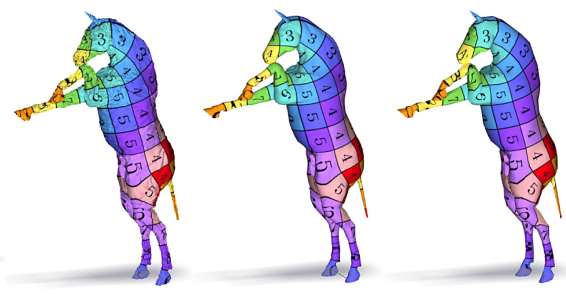

Noisy Vertex Topological Noise Sampled

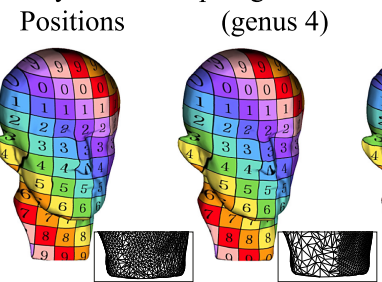
( $5 \%$ \#vertices $)$

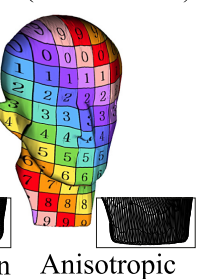

Fig. 12. Robustness to noise and sampling. Top row: a shape with various transformations from SHREC'10 (Bronstein et al. 2010). Bottom row: the left shape is mapped to the same geometry with different tessellations.

\subsection{Robustness}

We test our method on horse shapes from the SHREC'10 dataset (Bronstein et al. 2010), which contains various shapes with geometric and topological noise. The results are shown in the top row of Figure 12, where we compute a correspondence between the horse in a neutral pose (left) and a noisy pose: (center left) noise is added to the vertex positions; (center right) topological noise is introduced, e.g., between the rear feet; and (right) the mesh is sampled to $5 \%$ of the number of vertices. We initialized our method using 19 landmarks and obtained semantically correct results in all cases. The bottom row shows our results for different tessellations of a shape of a head, where one shape has uniform triangles, a second shape has larger triangles on one half and smaller triangles on the other half, and a third shape has highly anisotropic 

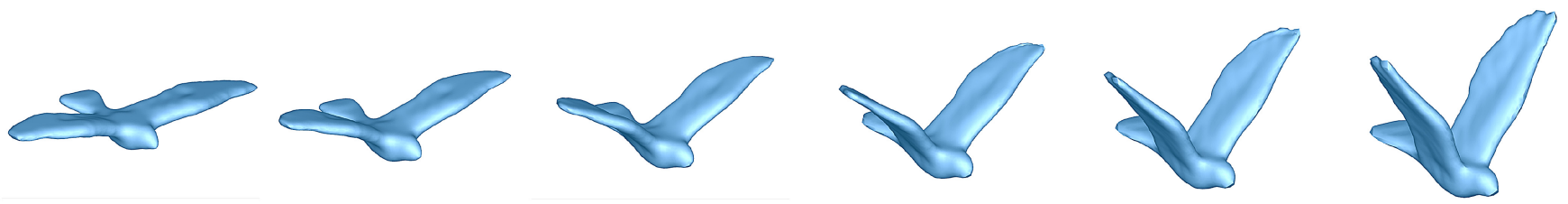

Fig. 13. Shape interpolation using our computed correspondence as input for Heeren et al. (2014).
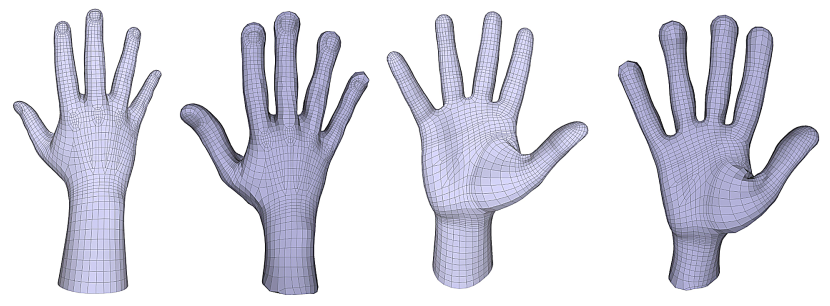

Fig. 14. Quad mesh transfer using our computed correspondence. Left: input quad mesh; right: output quad mesh. Note the preservation of the prominent edge flows in the quad mesh, such as the fingernails and knuckles.

triangles. For initialization, we used the identity map with additional noise. The resulting mapped texture is smooth and similar for the different tessellations.

\subsection{Application: Shape Interpolation}

Existing shape interpolation methods require the input shapes to share the same connectivity, while real data rarely satisfies this requirement. Our mapping can be used to remesh the target surface $M_{2}$ using the image of the vertices of $M_{1}$, given by $P_{12} V_{2}$, and the connectivity of $M_{1}$. We used our map between two birds from SHREC to demonstrate this application, starting from the BIM landmarks as described in Section 5.2. After remeshing $M_{2}$, as a few faces had a zero area, we iteratively moved vertices of the degenerate faces to an average of their one-ring neighbors until there were no degenerate faces. We then used the shape interpolation method by Heeren et al. (2014) to interpolate between the shapes, as shown in Figure 13. Note that we correctly mapped the wings, head, and tail of the birds, as is evident from the natural interpolation results.

\subsection{Application: Quad Mesh Transfer}

Finally, we demonstrate a potential application of our correspondence to quad mesh transfer of artist-generated quad meshes to scanned meshes. In this experiment, we start from a set of 41 landmark points and use weak landmark constraints with $\gamma=5 \cdot 10^{-5}$. We choose to have a larger number of landmarks in this example to preserve the fine features such as fingernails and joints. The results are shown in Figure 14, with two views of the input and output quad meshes on the left and right, respectively. Note that the edge flow of the output quad mesh closely follows the features of the hand, and the special structures in the input, such as the fingernails and knuckles, are nicely preserved in the output mesh. Such a high-quality transfer can only be achieved if the computed map has a low conformal distortion, which leads to well-preserved quad shapes.

\section{CONCLUSION}

We have suggested a novel correspondence method between nonisometric shapes, which considerably improves conformal distortion over existing techniques while preserving semantic features. Our approach is based on an alternating minimization of a quadratic energy, which is efficient, easy to implement, and flexible. In addition to demonstrating effectiveness on various benchmarks, we have shown applications to quad mesh transfer and shape interpolation.

Beyond its current utility, our formulation can serve as a framework for more involved energies on the differential of the map. For example, one can consider various metrics, such as nonisotropic, higher order, sparsity based, and data driven. We hope that our approach can be a stepping stone to generalizing the large body of literature on planar shape parameterization to the more general setting of shape correspondence.

\section{APPENDIX A REVERSIBILITY}

Proposition 1. Let $\left(M_{1}, g_{1}\right),\left(M_{2}, g_{2}\right)$ be two smooth compact Riemannian surfaces, with geodesic distance functions $d_{M_{i}}(\cdot, \cdot)$ given by the metrics $g_{i}$, respectively, and let $\phi_{12}: M_{1} \rightarrow M_{2}, \phi_{21}: M_{2} \rightarrow M_{1}$ be smooth maps. If there exists $\epsilon \geq 0$ such that

$$
d_{M_{1}}\left(p_{1}, \phi_{21}\left(\phi_{12}\left(p_{1}\right)\right)\right) \leq \epsilon, \quad \forall p_{1} \in M_{1},
$$

then:

(1) $\phi_{12}\left(p_{1}\right)=\phi_{12}\left(q_{1}\right) \Rightarrow d_{M_{1}}\left(p_{1}, q_{1}\right) \leq 2 \epsilon, \quad \forall p_{1}, q_{1} \in M_{1}$, and

(2) $\forall p_{1} \in M_{1} \exists p_{2} \in M_{2} \quad$ s.t. $\quad d_{M_{1}}\left(p_{1}, \phi_{21}\left(p_{2}\right)\right) \leq \epsilon$.

As a corollary of Proposition 1 we have that if the reversibility energy defined in Equation (6) is zero, then the maps $\phi_{12}, \phi_{21}$ are both injective and surjective.

Proof. Let $p_{1}, q_{1} \in M_{1}$, and set $p_{2}=\phi_{12}\left(p_{1}\right), q_{2}=\phi_{12}\left(q_{1}\right)$. Further, set $\hat{p}_{1}=\phi_{21}\left(p_{2}\right)$, and $\hat{q}_{1}=\phi_{21}\left(q_{2}\right)$.

(1) From the triangle inequality we have that $d_{M_{1}}\left(p_{1}, q_{1}\right) \leq$ $d_{M_{1}}\left(p_{1}, \hat{q}_{1}\right)+d_{M_{1}}\left(\hat{q}_{1}, q_{1}\right)$. From the assumption (1) we have that $p_{2}=q_{2}$ and therefore $\hat{p}_{1}=\hat{q}_{1}$. Thus, we have $d_{M_{1}}\left(p_{1}\right.$, $\left.\hat{q}_{1}\right)=d_{M_{1}}\left(p_{1}, \hat{p}_{1}\right) \leq \epsilon$ and $d_{M_{1}}\left(\hat{q}_{1}, q_{1}\right) \leq \epsilon$, which gives the required result.

(2) This follows trivially from the assumption of the Proposition if we set $p_{2}=\phi_{12}\left(p_{1}\right)$.

\section{REFERENCES}

Noam Aigerman, Shahar Z. Kovalsky, and Yaron Lipman. 2017. Spherical orbifold Tutte embeddings. ACM Transactions on Graphics (TOG) 36, 4 (2017), 90.

Noam Aigerman and Yaron Lipman. 2015. Orbifold Tutte embeddings. ACM Transactions on Graphics (TOG) 34 (2015), 190:1-190:12.

Noam Aigerman and Yaron Lipman. 2016. Hyperbolic orbifold Tutte embeddings. ACM Transactions on Graphics (TOG) 35 (2016), 217:1-217:14.

Noam Aigerman, Roi Poranne, and Yaron Lipman. 2015. Seamless surface mappings. ACM Transactions on Graphics (TOG) 34, 4 (2015), 72. 
Mathieu Aubry, Ulrich Schlickewei, and Daniel Cremers. 2011. The wave kernel signature: A quantum mechanical approach to shape analysis. In International Conference on Computer Vision Workshops (ICCV Workshops'11). IEEE.

Alexander M. Bronstein, Michael M. Bronstein, Benjamin Bustos, Umberto Castellani, Marco Crisani, Bianca Falcidieno, Leonidas J. Guibas, Iasonas Kokkinos, Vittorio Murino, Maks Ovsjanikov, Giuseppe Patane, Michuela Spagnuolo, and Jian Sun 2010. SHREC 2010: Robust feature detection and description benchmark. Proceedings of 3DOR 2, 5 (2010), 6.

Alexander M. Bronstein, Michael M. Bronstein, and Ron Kimmel. 2006. Generalized multidimensional scaling: A framework for isometry-invariant partial surface matching. Proceedings of the National Academy of Sciences 103, 5 (2006), 11681172 .

Oliver Burghard, Alexander Dieckmann, and Reinhard Klein. 2017. Embedding shapes with Green's functions for global shape matching. Computers \& Graphics 68 (2017), 1-10.

Isaac Chao, Ulrich Pinkall, Patrick Sanan, and Peter Schröder. 2010. A simple geometric model for elastic deformations. ACM Transactions on Graphics (TOG) 29, 4 (2010), 38.

Xiaobai Chen, Aleksey Golovinskiy, and Thomas Funkhouser. 2009. A benchmark for 3D mesh segmentation. ACM Transactions on Graphics (Proceedings of SIGGRAPH) 28, 3 (Aug. 2009), 73:1-73:12.

Xiaobai Chen, Abulhair Saparov, Bill Pang, and Thomas Funkhouser. 2012. Schelling points on 3D surface meshes. ACM Transactions on Graphics (TOG) 31, 4 (2012), 29.

Trevor F. Cox and Michael A. A. Cox. 2000. Multidimensional Scaling. CRC Press.

James Eells and Joseph H. Sampson. 1964. Harmonic mappings of Riemannian manifolds. American fournal of Mathematics 86, 1 (1964), 109-160.

Danielle Ezuz and Mirela Ben-Chen. 2017. Deblurring and denoising of maps between shapes. In Computer Graphics Forum, Vol. 36. 165-174.

Donald Geman and Chengda Yang. 1995. Nonlinear image recovery with halfquadratic regularization. IEEE Transactions on Image Processing 4, 7 (1995), 932946.

Daniela Giorgi, Silvia Biasotti, and Laura Paraboschi. 2007. SHREC: Shape Retrieval Contest: Watertight Models Track

Xianfeng Gu, Yalin Wang, Tony F. Chan, Paul M. Thompson, and Shing-Tung Yau. 2004. Genus zero surface conformal mapping and its application to brain surface mapping. Transactions on Medical Imaging 23, 8 (2004), 949-958.

Behrend Heeren, Martin Rumpf, Peter Schröder, Max Wardetzky, and Benedikt Wirth 2014. Exploring the geometry of the space of shells. Computer Graphics Forum 33, 5 (2014), 247-256.

Behrend Heeren, Martin Rumpf, Max Wardetzky, and Benedikt Wirth. 2012. Timediscrete geodesics in the space of shells. Computer Graphics Forum 31 (2012), 17551764.

Kai Hormann and Günther Greiner. 2000. MIPS: An Efficient Global Parametrization Method. Technical Report. DTIC Document.

Ruqi Huang and Maks Ovsjanikov. 2017. Adjoint map representation for shape analysis and matching. In Computer Graphics Forum, Vol. 36. 151-163.

Hiroyasu Izeki and Shin Nayatani. 2005. Combinatorial harmonic maps and discretegroup actions on Hadamard spaces. Geometriae Dedicata 114, 1 (2005), 147-188.

Evangelos Kalogerakis, Aaron Hertzmann, and Karan Singh. 2010. Learning 3D mesh segmentation and labeling. ACM Transactions on Graphics 29, 3 (2010), 102:1102:12.

Vladimir G. Kim, Yaron Lipman, and Thomas Funkhouser. 2011. Blended intrinsic maps. ACM Transactions on Graphics (TOG) 30 (2011), 79:1-79:12.

Artiom Kovnatsky, Michael M. Bronstein, Xavier Bresson, and Pierre Vandergheynst. 2015. Functional correspondence by matrix completion. In Proceedings of Computer Vision and Pattern Recognition (CVPR'15).

Artiom Kovnatsky, Michael M. Bronstein, Alexander M. Bronstein, Klaus Glashoff, and Ron Kimmel. 2013. Coupled quasi-harmonic bases. Computer Graphics Forum 32 (2013), 439-448.

Zorah Lähner, Matthias Vestner, Amit Boyarski, Or Litany, Ron Slossberg, Tal Remez, Emanuele Rodolà, Alexander M. Bronstein, Michael M. Bronstein, Ron Kimmel, and Daniel Cremers. 2017. Efficient deformable shape correspondence via kernel matching. In $3 D$ Vision (3DV'17)

Bruno Lévy, Sylvain Petitjean, Nicolas Ray, and Jérome Maillot. 2002. Least squares conformal maps for automatic texture atlas generation. In ACM Transactions on Graphics (TOG), Vol. 21. ACM, 362-371.

Manish Mandad, David Cohen-Steiner, Leif Kobbelt, Pierre Alliez, and Mathieu Desbrun. 2017. Variance-minimizing transport plans for inter-surface mapping. ACM Transactions on Graphics 36 (2017), 14

Haggai Maron, Nadav Dym, Itay Kezurer, Shahar Kovalsky, and Yaron Lipman. 2016. Point registration via efficient convex relaxation. ACM Transactions on Graphics (TOG) 35 (2016), 73:1-73:12.
Brent C. Munsell, Pahal Dalal, and Song Wang. 2008. Evaluating shape correspondence for statistical shape analysis: A benchmark study. IEEE Transactions on Pattern Analysis and Machine Intelligence 30, 11 (2008), 2023-2039.

Seiki Nishikawa. 2000. Variational Problems in Geometry. AMS.

Dorian Nogneng and Maks Ovsjanikov. 2017. Informative descriptor preservation via commutativity for shape matching. In Computer Graphics Forum, Vol. 36. 259-267.

Maks Ovsjanikov, Mirela Ben-Chen, Justin Solomon, Adrian Butscher, and Leonidas Guibas. 2012. Functional maps: A flexible representation of maps between shapes. ACM Transactions on Graphics (TOG) 31 (2012), 30:1-30:11.

Daniele Panozzo, Ilya Baran, Olga Diamanti, and Olga Sorkine-Hornung. 2013. Weighted averages on surfaces. ACM Transactions on Graphics (TOG) 32, 4 (2013), 60.

Ulrich Pinkall and Konrad Polthier. 1993. Computing discrete minimal surfaces and their conjugates. Experimental Mathematics 2, 1 (1993), 15-36.

Emanuele Rodolà, Michael Moeller, and Daniel Cremers. 2015. Point-wise map recovery and refinement from functional correspondence. In Proceedings of Vision, Modeling and Visualization (VMV'15).

Y. Sahillioğlu and Yücel Yemez. 2011. Coarse-to-fine combinatorial matching for dense isometric shape correspondence. In Computer Graphics Forum, Vol. 30 1461-1470.

Matan Sela, Yonathan Aflalo, and Ron Kimmel. 2015. Computational caricaturization of surfaces. Computer Vision and Image Understanding 141 (2015), 1-17.

Rui Shi, Wei Zeng, Zhengyu Su, Jian Jiang, Hanna Damasio, Zhonglin Lu, Yalin Wang Shing-Tung Yau, and Xianfeng Gu. 2017. Hyperbolic harmonic mapping for surface registration. IEEE Transactions on Pattern Analysis and Machine Intelligence 39,5 (2017), 965-980.

Alon Shtern and Ron Kimmel. 2014. Iterative closest spectral kernel maps. In 3D Vision (3DV'14). IEEE.

Justin Solomon, Gabriel Peyré, Vladimir G. Kim, and Suvrit Sra. 2016. Entropic metric alignment for correspondence problems. ACM Transactions on Graphics (TOG) 35 (2016), 72:1-72:13.

Olga Sorkine and Marc Alexa. 2007. As-rigid-as-possible surface modeling. In Computer Graphics Forum, Vol. 4.

Robert W. Sumner and Jovan Popović. 2004. Deformation transfer for triangle meshes. ACM Transactions on Graphics (TOG) 23 (2004), 399-405.

Gary K. L. Tam, Zhi-Quan Cheng, Yu-Kun Lai, Frank C. Langbein, Yonghuai Liu, David Marshall, Ralph R. Martin, Xian-Fang Sun, and Paul L. Rosin. 2013. Registration of 3D point clouds and meshes: A survey from rigid to nonrigid. IEEE Transactions on Visualization and Computer Graphics 19, 7 (2013), 1199-1217.

Alex Tsui, Devin Fenton, Phong Vuong, Joel Hass, Patrice Koehl, Nina Amenta, David Coeurjolly, Charles DeCarli, and Owen Carmichael. 2013. Globally optimal cortical surface matching with exact landmark correspondence. In Information Processing in Medical Imaging, Vol. 23. NIH Public Access, 487.

Hajime Urakawa. 1993. Calculus of Variations and Harmonic Maps. AMS.

Oliver Van Kaick, Hao Zhang, Ghassan Hamarneh, and Daniel Cohen-Or. 2011. A survey on shape correspondence. In Computer Graphics Forum, Vol. 30 1681-1707.

Matthias Vestner, Roee Litman, Emanuele Rodolà, Alex Bronstein, and Daniel Cremers. 2017. Product manifold filter: Non-rigid shape correspondence via kernel density estimation in the product space. In Proceedings of Computer Vision and Pattern Recognition (CVPR'17). 3327-3336.

Christoph Von-Tycowicz, Christian Schulz, Hans-Peter Seidel, and Klaus Hildebrandt. 2015. Real-time nonlinear shape interpolation. ACM Transactions on Graphics (TOG) 34 (2015), 34:1-34:10.

Yilun Wang, Junfeng Yang, Wotao Yin, and Yin Zhang. 2008. A new alternating minimization algorithm for total variation image reconstruction. SIAM fournal on Imaging Sciences 1, 3 (2008), 248-272.

Kai Xu, Vladimir G. Kim, Qixing Huang, Niloy Mitra, and Evangelos Kalogerakis. 2016. Data-driven shape analysis and processing. In SIGGRAPH ASIA 2016 Courses. ACM, 4.

Yangyang Xu and Wotao Yin. 2013. A block coordinate descent method for regularized multiconvex optimization with applications to nonnegative tensor factorization and completion. SIAM fournal on Imaging Sciences 6, 3 (2013), 1758-1789.

Xiaopeng Zheng, Chengfeng Wen, Na Lei, Ming Ma, and Xianfeng Gu. 2017. Surface registration via foliation. In Proceedings of the IEEE Conference on Computer Vision and Pattern Recognition. 938-947.

Daniel Zoran and Yair Weiss. 2011. From learning models of natural image patches to whole image restoration. In IEEE International Conference on Computer Vision (ICCV'11). IEEE, 479-486.

Received January 2018; revised October 2018; accepted January 2019 